\title{
THE SPACE OF SECTIONS OF A SPHERE-BUNDLE I
}

\author{
by M. C. CRABB and W. A. SUTHERLAND
}

(Received 10th June 1985)

\section{Introduction}

Throughout this paper $X$ will be a finite connected CW-complex of dimension $m$, and $\xi$ will be a real $(n+1)$-plane bundle over $X(n>0)$ equipped with a Riemannian metric. We aim to give a systematic account of the space $\Gamma S \xi$ of sections of the sphere-bundle $S \xi$.

In particular we prove a result, announced as Theorem 2.14 of [3], which shows that for $n>2 m+1$ the homotopy type of $\Gamma S \xi$ determines the stable homotopy type of the (stable) Thom space $X^{-\xi}$ (see Corollary 5.3 below). The ingredients of the proof are Freudenthal's suspension theorem over $X$ and $S$-duality theory.

In a sequel, we shall refine the Freudenthal theorem to an EHP sequence, in analogy with the classical procedure. This provides a natural setting for the results of [15] (see the remark on p. 224 of that paper).

The later sections of the paper deal with the homology of $\Gamma S \xi$. Let $\mathscr{H}$ be the group of orientation-preserving isometric isomorphisms of $\xi$ over $X$ and $P \rightarrow B$ a principal $\mathscr{H}$ bundle over a finite $C W$-complex $B$. The main result is a computation of the homology $H_{j}\left(P \times{ }_{\mathscr{H}} \Gamma S \xi\right)$, up to group extension, for $j<2 n-2 m-1$ (see Corollary 9.4), by a sort of "Gysin sequence" involving $B$ and $X^{-\xi}$. As an application we prove two results of J. M. Møller, [11], [12] and [13], on the homology of spaces of sections of projective bundles.

- The detailed statements of our results appear in the relevant sections, according to the following plan. In Section 2 we summarize some facts about the geometry of $\Gamma S \xi$. In Section 3, assuming that $\Gamma S \xi$ is non-empty, we obtain preliminary results on its homotopy; in particular we show that each component is a space of finite type. In Section 4 we establish our basic notation before describing the Freudenthal suspension theorem. Duality is discussed in Section 5, and under suitable restrictions this adds precision to the description in Section 3 of the components of $\Gamma S \xi$ (Theorem 5.1). In Section 6 the suspension process is reformulated in two ways using the stable cohomotopy Euler class. The first of these is used in Section 7 to study the action of certain symmetries of $S \xi$ on the components of $\Gamma S \xi$. Finally, in Sections 8 and 9 we apply previous work to get information on the homology of $\Gamma S \xi$.

At two stages we append clarifying details. The appendix to Section 4 deals with "the Thom space of a virtual bundle", and the appendix to Section 9 on "stable homotopy over a base space" includes the proof of a result (essentially standard, but for which we know of no suitable reference) used in that section. 


\section{Geometry of $\Gamma S \xi$}

We topologize $\Gamma S \xi$ as a subspace of the Banach space $\Gamma \xi$ of sections of the vector bundle $\xi$. Then $\Gamma S \xi$ is a smooth submanifold. Its tangent space at a point $s$ of $\Gamma S \xi$ is $\Gamma \zeta(s)$, where $\zeta(s)$ is the sub-bundle of $\xi$ which is fibrewise orthogonal to the onedimensional sub-bundle spanned by $s$. The normal bundle of $\Gamma S \xi$ in $\Gamma \xi$ is trivial, with fibre the space $\mathscr{C}(X)$ of continuous real-valued functions on $X$.

In fact $\Gamma S \xi$ even has an algebraic structure. To see this, let us write $A$ for the commutative ring $\mathscr{C}(X)$ and $P$ for the finitely-generated projective $A$-module $\Gamma \xi$. The Riemannian metric on $\xi$ induces a non-singular bilinear form $\langle\rangle:, P \times P \rightarrow A$. Our space $\Gamma S \xi$ is the set $\{s \in P \mid\langle s, s\rangle=1\}$. It is now easy to see that $\Gamma S \xi$ is an affine algebraic variety over $A$.

Let $\mathscr{G}$ be the group of isometric automorphisms of $\xi$ over $X$. Then $\mathscr{G}$ is a Lie group (in general infinite-dimensional) which is algebraic over $A$ and acts smoothly on $\Gamma S \xi$. It is easy to show that each component of $\Gamma S \xi$ is a homogeneous space of the connected component $\mathscr{G}^{0}$ of the identity in $\mathscr{G}$. The part of this assertion which we need later is recorded in:

Proposition 2.1. The group $\mathscr{G}^{0}$ acts transitively on each component of $\Gamma S \xi$.

Proof. See (5.3) of [3].

\section{Homotopy type of $\Gamma S \xi$}

Throughout the rest of the paper we assume that $\Gamma S \xi$ is non-empty, and we fix a section $s_{0}$ in $\Gamma S \xi$. We shall study the homotopy type of the pointed space $\left(\Gamma S \xi, s_{0}\right)$. By (2.1) this homotopy type depends only on the homotopy class of $s_{0}$.

For convenience we often adopt a slightly different viewpoint on $\left(\Gamma S \xi, s_{0}\right)$, as we shall now describe. Throughout the paper we use the same symbol for a vector space $V$ and the product bundle with fibre $V$ over any space. With this notation, $s_{0}$ determines an orthogonal splitting $\xi=\zeta \oplus \mathbb{R}$, where $\zeta$ is an $n$-plane bundle over $X$ and $s_{0}$ is the constant section of $\mathbb{R}$ with value 1 . Now $S \xi$ may be canonically identified with the fibrewise one-point compactification $\zeta^{+}$of $\zeta$ (obtained by adjoining a basepoint at infinity to each fibre of $\zeta$ ), in such a way that $s_{0}$ corresponds to the section $s_{+}$of $\zeta^{+}$ which picks out the basepoint in each fibre. We often state results in terms of the pointed space $\Gamma \zeta^{+}$, with the basepoint $s_{+}$understood. These results may of course be translated into corresponding results about $\left(\Gamma S \xi, s_{0}\right)$.

Proposition 3.1. (a) The fundamental group $\pi_{1}\left(\Gamma \zeta^{+}\right)$is finitely generated nilpotent.

(b) If $r>1$ then $\pi_{r}\left(\Gamma \zeta^{+}\right)$is finitely generated abelian.

(c) If $0 \leqq r<n-m$ then $\pi_{r}\left(\Gamma \zeta^{+}\right)=0$.

Most of this proposition is a special case of a result for locally trivial bundles with nilpotent fibres (cf. [7] II.2). We include a proof for completeness.

Proof. We use induction over the cells of $X$. The result holds when $X$ is a single point, for then $m=0$ and $\Gamma \zeta^{+}$is an $n$-sphere $S^{n}$. 
Suppose that the proposition holds for $(n+1)$-plane bundles over a finite connected CW-complex $Y$ with $\operatorname{dim} Y \leqq m$, and that $X$ is obtained from $Y$ by attaching a single $m$-cell with characteristic map $f:\left(D^{m}, S^{m-1}\right) \rightarrow(X, Y)$. Let $\Gamma\left(Y ; \zeta^{+}\right)$denote the pointed space of sections of the restriction $\zeta^{+} \mid Y$. When different base spaces are involved, as here, we shall sometimes for clarity write $\Gamma\left(X ; \zeta^{+}\right)$instead of $\Gamma \zeta^{+}$. Since the inclusion of $Y$ in $X$ is a cofibration, the restriction map from $\Gamma\left(X ; \zeta^{+}\right)$to $\Gamma\left(Y ; \zeta^{+}\right)$is a fibration. We write $\Gamma\left(X, Y ; \zeta^{+}\right)$for the fibre over $s_{+}$. There is a commutative diagram of fibrations:

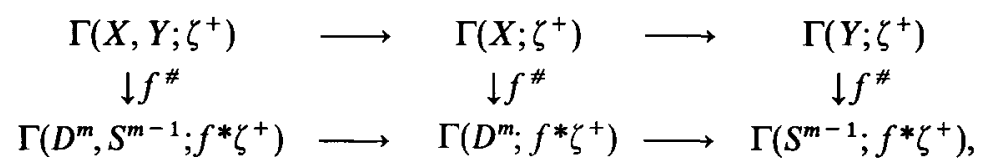

and the map $f^{\#}$ on fibres is a homeomorphism. After choosing a trivialization of $f^{*} \zeta^{+}$ over $D^{m}$, we may identify the fibre with the iterated loop space $\Omega^{m} S^{n}$. From the homotopy exact sequence of the upper fibration in (3.2), it is now easy to see that the assertions of the proposition hold for $X$ provided either $n-m>1$ or we restrict attention to those assertions concerning $r>1$. The fundamental group requires a little more care when $n-m \leqq 1$, and we deal with it next.

Recall that whenever $F \stackrel{i}{\longrightarrow} E \stackrel{p}{\longrightarrow} B$ is a fibration there is a natural action (indicated here by a dot) of $\pi_{1} E$ on $\pi_{1} F$, such that for any $g, h$ in $\pi_{1} E, \pi_{1} F$ we have $i_{*}(g \cdot h)=$ $g i_{*}(h) g^{-1}$. To compute this action at the upper level in (3.2), by naturality we may work at the lower level. But there the action of $\pi_{1}\left(S^{n}\right)$ on $\pi_{1}\left(\Omega^{m} S^{n}\right)$ is the standard action on $\pi_{m+1}\left(S^{n}\right)$, which is trivial. Thus, writing $\pi$ now for $\pi_{1} \Gamma\left(X ; \zeta^{+}\right)$, we see that $\pi$ is a central extension

$$
0 \rightarrow N \rightarrow \pi \rightarrow H \rightarrow 0
$$

in which $N$ is a quotient of $\pi_{1}\left(\Omega^{m} S^{n}\right)$ and hence is finitely generated abelian, while $H$ is a subgroup of $\pi_{1} \Gamma\left(Y ; \zeta^{+}\right)$. By inductive hypothesis $\pi_{1} \Gamma\left(Y ; \zeta^{+}\right)$is finitely generated nilpotent, hence so is $H$ and by (3.3) so is $\pi$. This completes the inductive step.

We write $\left(\Gamma \zeta^{+}\right)^{0}$ for the component of $s_{+}$in $\Gamma \zeta^{+}$. From now on we write $a_{0}, b_{0}, \ldots$ for the basepoints of pointed spaces $A, B, \ldots$.

Proposition 3.4. The pointed space $\left(\Gamma \zeta^{+}\right)^{0}$ is homotopy equivalent to a pointed $\mathrm{CW}$ complex $A$ with finite skeleta $A^{(r)}$ for all $r \geqq 0$ and $A^{(r)}=\left\{a_{0}\right\}$ for $r<n-m$.

Proof. We use the finiteness criterion of Wall [16]. By $(3.1), \pi_{1}\left(\Gamma \zeta^{+}\right)^{0}$ is finitely generated nilpotent. Hence it is finitely presentable and (by Hilbert's basis theorem) its integral group ring is Noetherian. Since $\left(\Gamma \zeta^{+}\right)^{0}$ is locally path-connected and semilocally simply-connected it has a universal cover. The homotopy groups and hence the homology groups of the universal cover are finitely generated as abelian groups (again by (3.1)) and a fortiori as modules over the group ring. We may therefore apply Wall's construction, beginning with $A^{(n-m-1)}=\left\{a_{0}\right\}$ if $m<n$, and get $A$ as specified in the proposition together with a weak homotopy equivalence $f: A \rightarrow\left(\Gamma \zeta^{+}\right)^{0}$. Finally, $\left(\Gamma \zeta^{+}\right)^{0}$ has the homotopy type of a CW-complex by Milnor [10] (cf. Lemma 8.5 of [3]), so $f$ is a homotopy equivalence. 


\section{Stabilization}

In this section we describe the suspension theorem mentioned in the introduction, and prepare the way for the discussion of duality in Section 5. As in Section 3 we use the $\zeta^{+}$ viewpoint. We shall be doing homotopy theory over the base space $X$; [9] is a good textbook reference for the basic theory. First we must fix some notation.

Let $Q_{0} \rightarrow B, Q_{1} \rightarrow B$ be locally trivial bundles of pointed finite $\mathrm{CW}$-complexes over a fixed finite CW-complex $B$. (This is sufficiently general for our purposes and indeed we shall be concerned mainly with sphere-bundles.) In the terminology of [9] these are (well) sectioned spaces over $B$ (with sections given by the basepoints in the fibres). We write $\left[Q_{0} ; Q_{1}\right]_{B}$ for the set of homotopy classes of fibrewise pointed maps from $Q_{0}$ to $Q_{1}$ over $B$. When $A$ is a subcomplex of $B$ we write $\left[Q_{0} ; Q_{1}\right]_{(B, A)}$ for the corresponding set in which each map takes the fibre over any point of $A$ to the appropriate basepoint.

For example if 0 is the zero vector bundle over $X$ then $\left[0^{+} ; \zeta^{+}\right]_{X}$ is $\pi_{0} \Gamma \zeta^{+}$, since a fibrewise pointed map $0^{+} \rightarrow \zeta^{+}$is determined by its restriction to 0 and this is simply a section of $\zeta^{+}$. Similarly $\pi_{0} \Gamma\left(X, Y ; \zeta^{+}\right)=\left[0^{+} ; \zeta^{+}\right]_{(X, Y)}$ where $\Gamma\left(X, Y ; \zeta^{+}\right)$is as in (3.2).

Now we stabilize $\left[Q_{0} ; Q_{1}\right]_{B}$. First, we have a map from

$$
\left[Q_{0} ; Q_{1}\right]_{B} \text { to }\left[\mathbb{R}^{+} \wedge{ }_{B} Q_{0} ; \mathbb{R}^{+} \wedge{ }_{B} Q_{1}\right]_{B}
$$

given by the smash product (over $B$ ) with the identity map of the trivial bundle $\mathbb{R}^{+}$. Iterating this process (and making the standard identification of the $k$-fold product $\mathbb{R}^{+} \wedge \cdots \wedge \mathbb{R}^{+}$with $\left.\left(\mathbb{R}^{k}\right)^{+}\right)$, we define the stable group $\omega_{B}^{0}\left\{Q_{0} ; Q_{1}\right\}$ as the direct limit of the sets $\left[\left(\mathbb{R}^{k}\right)^{+} \wedge_{B} Q_{0} ;\left(\mathbb{R}^{k}\right)^{+} \wedge_{B} Q_{1}\right]_{B}(k \geqq 0)$. Its elements are called stable maps $Q_{0} \rightarrow Q_{1}$ over $B$. The stabilization map from $\left[Q_{0} ; Q_{1}\right]_{B}$ to $\omega_{B}^{0}\left\{Q_{0} ; Q_{1}\right\}$ will be denoted by $E$. The relative group $\omega_{(B, A)}^{0}\left\{Q_{0} ; Q_{1}\right\}$ is defined similarly and again we write $E$ for stabilization.

In the usual way we introduce groups $\omega_{B}^{j}\left\{Q_{0} ; Q_{1}\right\}$ for $j \in \mathbb{Z}$ as the direct limit of the sets $\left[\left(\mathbb{R}^{k}\right)^{+}{ }_{{ }_{B}} Q_{0} ;\left(\mathbb{R}^{l}\right)^{+} \wedge_{B} Q_{1}\right]_{B}(k, l \geqq 0, l-k=j)$. Relative groups are defined similarly, and there is a long exact sequence relating $\omega_{(B, A)}^{*}\left\{Q_{0} ; Q_{1}\right\}, \omega_{B}^{*}\left\{Q_{0} ; Q_{1}\right\}$ and $\omega_{A}^{*}\left\{Q_{0}\left|A ; Q_{1}\right| A\right\}$.

If $B$ is a point then we omit it from the notation and we are doing standard stable homotopy. So if $Z_{0}, Z_{1}$ are pointed finite $\mathrm{CW}$-complexes we write $\omega^{0}\left\{Z_{0} ; Z_{1}\right\}$, rather than the more usual $\left\{Z_{0} ; Z_{1}\right\}$, for the group of stable maps from $Z_{0}$ to $Z_{1}$. Later we shall introduce analogous groups with stable homotopy $\omega$ replaced by homology $H$. When we use $\omega$ and $H$ in the usual way (for example $\omega^{0}(X)$ ) we mean the unreduced theories; $\tilde{\omega}$ and $\tilde{H}$ denote the reduced theories. Thus : $\omega^{j}\left\{Z_{0} ; S^{0}\right\}=\tilde{\omega}^{j}\left(Z_{0}\right), \omega^{j}\left\{S^{0} ; Z_{1}\right\}=$ $\tilde{\omega}_{-j}\left(Z_{1}\right)$.

We are interested in sphere bundles over $X$. Let $\alpha_{0}, \alpha_{1}$ be real vector bundles over $X$. Then we may think of the group $\omega_{X}^{0}\left\{\alpha_{0}^{+} ; \alpha_{1}^{+}\right\}$of stable maps of sphere-bundles over $X$ as stable cohomotopy groups of Thom spaces. We use the traditional notation $X^{\xi}$ for the Thom space of $\xi$, and write $(X, Y)^{\xi}$ for the quotient of $X^{\xi}$ by $Y^{\xi \mid Y}$. As we describe in detail in the appendix to this section, we may extend this notation to the case of the virtual vector bundle $\alpha=\alpha_{0}-\alpha_{1}$ over $X$. We have then:

$$
\begin{aligned}
\omega_{X}^{0}\left\{\alpha_{0}^{+} ; \alpha_{1}^{+}\right\} & =\tilde{\omega}^{0}\left(X^{\alpha}\right) ; \\
\omega_{(X, Y)}^{0}\left\{\alpha_{0}^{+} ; \alpha_{1}^{+}\right\} & =\tilde{\omega}^{0}\left((X, Y)^{\alpha}\right) .
\end{aligned}
$$

We shall use the following local coefficient notation for any well-behaved cohomology 
theory $h$, such as integral cohomology or stable cohomotopy:

$$
\begin{gathered}
h^{j}(X ; \alpha)=\tilde{h}^{j}\left(X^{\alpha}\right) \\
h^{j}(X, Y ; \alpha)=\tilde{h}^{j}\left((X, Y)^{\alpha}\right) .
\end{gathered}
$$

(Again, details are in the appendix). If $\alpha$ is zero, then $h^{j}(X ; 0)$ is simply $h^{j}(X)$.

By (4.1) and (4.2) we can now write stabilization as $E:\left[\alpha_{0}^{+} ; \alpha_{1}^{+}\right]_{X} \rightarrow \omega^{0}(X ; \alpha)$.

Since $\left[0^{+} ; \zeta^{+}\right]_{X}=\pi_{0} \Gamma \zeta^{+}$, we have in particular a stabilization map

$$
E: \pi_{0} \Gamma \zeta^{+} \rightarrow \omega^{0}(X ;-\zeta)
$$

and similarly in the relative case.

Theorem 4.3. The stabilization maps

(a) $E: \pi_{0} \Gamma \zeta^{+} \rightarrow \omega^{0}(X ;-\zeta)$ and

(b) $E: \pi_{0} \Gamma\left(X, Y ; \zeta^{+}\right) \rightarrow \omega^{0}(X, Y ;-\zeta)$

are bijective if $m<2 n-1$, surjective if $m \leqq 2 n-1$.

This may readily be deduced from the following more precise result. If $s \in \Gamma \zeta^{+}$we shall sometimes write $E(s) \in \omega^{\circ}(X ;-\zeta)$ for the stabilization of the class represented by $s$.

Theorem 4.4. Let $m \leqq 2 n-1$. Suppose that $x$ is an element of $\omega_{X}^{0}\left\{0^{+} ; \zeta^{+}\right\}$and $s$ a section of $\zeta^{+} \mid Y$ such that $x$ restricts to $E(s)$ in $\omega_{Y}^{0}\left\{0^{+} ; \zeta^{+} \mid Y\right\}$. Then there is a section $\tilde{s}$ in $\Gamma\left(X ; \zeta^{+}\right)$extending $s$ and such that $E(\tilde{s})=x$.

The proof of (4.4) is by induction over the cells of $X$. At the inductive step one uses Freudenthal's suspension theorem for homotopy groups of spheres. We omit the details.

We can obtain information on $\Gamma \zeta^{+}$from (4.3) by simply taking adjoints. Let $Z$ be a finite pointed CW-complex and let us denote the pull-back of $\zeta^{+}$under the projection of $Z \times X$ onto $X$ again by $\zeta^{+}$. Taking adjoints, we may identify the space of pointed maps from $Z$ to $\Gamma\left(X ; \zeta^{+}\right)$with $\Gamma\left(\left(Z, z_{0}\right) \times X ; \zeta^{+}\right)$. Hence, as in (4.3)(b), we have a stabilization map

$$
E:\left[Z ; \Gamma\left(X ; \zeta^{+}\right)\right]=\pi_{0} \Gamma\left(\left(Z, z_{0}\right) \times X ; \zeta^{+}\right) \rightarrow \omega^{0}\left(\left(Z, z_{0}\right) \times X ;-\zeta\right)
$$

An immediate corollary of $(4.3)(b)$ is:

Corollary 4.6. The map $E$ in (4.5) is bijective if $m+\operatorname{dim} Z<2 n-1$, surjective if $m+\operatorname{dim} Z \leqq$ $2 n-1$.

When $Z=S^{j}$ the righthand group in (4.5) is $\omega^{-j}(X ;-\zeta)$, and we get:

Corollary 4.7. The stabilization map

$$
E: \pi_{j}\left(\Gamma \zeta^{+}\right) \rightarrow \omega^{-j}(X ;-\zeta)
$$

is bijective if $j<2 n-m-1$, surjective if $j \leqq 2 n-m-1$. 
Remark 4.8. In particular $\pi_{1} \Gamma \zeta^{+}$is abelian when $m<2 n-2$. For $E$ in (4.7) is clearly a group homomorphism if $j>0$. If $m<2 n-2$ then (4.7) applies with $j=1$ and $\pi_{1} \Gamma \zeta^{+}$ is isomorphic to the abelian group $\omega^{-1}(X ;-\zeta)$.

\section{Appendix: the Thom space of a virtual bundle}

A virtual bundle $\alpha$ over $X$ is just an ordered pair $\left(\alpha_{0}, \alpha_{1}\right)$ of real vector bundles; it is often written as $\alpha_{0}-\alpha_{1}$. We want to explain how a group such as $\left.\tilde{\omega}^{0} X^{\alpha}\right)$ is to be understood.

It may be helpful to recall first another, familiar, definition: that of the tangent space $T_{x} M$ at a point $x$ of a smooth $m$-manifold $M$. One procedure is as follows. We choose a chart $\phi: U \rightarrow V$, where $U \subseteq M$ is an open neighbourhood of $x$ and $V$ is an open subset of $\mathbb{R}^{m}$, and then for practical purposes take $T_{x} M$ to be the well understood tangent space $\mathbb{R}^{m}$ of $V$ at $\phi(x)$. Nevertheless $T_{x} M$ is a well-defined geometric object, and formally we can define a tangent vector at $x$ as an equivalence class of pairs $(\phi, v)$ where $\phi$ is a chart at $x$ and $v \in \mathbb{R}^{m}$.

To define $\tilde{h}^{j}\left(X^{\alpha}\right)$ we follow a similar recipe. Choose a trivialization $\phi: \alpha_{1} \oplus \sigma \rightarrow \mathbb{R}^{M}$ for some vector bundle $\sigma$ and $M \geqq 0$. Then take $\tilde{h}^{j}\left(X^{\alpha}\right)$ to be the cohomology $\tilde{h}^{j+M}\left(X^{\alpha_{0} \oplus \sigma}\right)$ of the genuine space $X^{\alpha_{0} \oplus \sigma}$, or in the notation (4.2) $h^{j+M}\left(X ; \alpha_{0} \oplus \sigma\right)$. To make sense of this as a definition we must say how the groups arising from different trivializations are to be identified. Let $\psi: \alpha_{1} \oplus \tau \rightarrow \mathbb{R}^{N}$ be a second trivialization. Consider first the case $\tau=\sigma \oplus \mathbb{R}, \quad N=M+1, \quad \psi=\phi \oplus 1$. Then we identify $h^{j+M}\left(X ; \alpha_{0} \oplus \sigma\right)$ with $h^{j+M+1}\left(X ; \alpha_{0} \oplus \sigma \oplus \mathbb{R}\right)=h^{j+N}\left(X ; \alpha_{0} \oplus \tau\right)$ by the suspension isomorphism. This allows us to stabilize, and in general we can reduce by repeated suspension isomorphisms to the situation in which $M$ and $N$ are equal and $\operatorname{dim} \sigma=\operatorname{dim} \tau$ is large compared with $\operatorname{dim} X$. Then the linear isomorphism $\psi^{-1} \circ \phi: \alpha_{1} \oplus \sigma \rightarrow \alpha_{1} \oplus \tau$ is homotopic to $1 \oplus f$ for some isomorphism $f: \sigma \rightarrow \tau$, and $f$ is unique up to homotopy. The isomorphism $1 \oplus f: \alpha_{0} \oplus \sigma \rightarrow \alpha_{0} \oplus \tau$ induces the required map $h^{j+N}\left(X ; \alpha_{0} \oplus \tau\right) \rightarrow h^{j+M}\left(X ; \alpha_{0} \oplus \sigma\right)$. This construction is consistent with stabilization, and so the identification is unambiguous.

Thus $\tilde{h}^{j}\left(X^{\alpha}\right)=h^{j}(X ; \alpha)$ is a well-defined abelian group, independent of any choices involved in its description. (Formally, an element is an equivalence class of pairs $(\phi, v)$ with $v \in h^{j+M}\left(X ; \alpha_{0} \oplus \sigma\right)$. And to avoid set-theoretic problems we had better insist that $\sigma$ is a sub-bundle of $\mathbb{R}^{M}$. But this formal approach does not add much to our understanding of $X^{\alpha}$.)

In the following sections we shall refer to the stable Thom space $X^{\alpha}$ itself. Our statements involving $X^{\alpha}$ can be interpreted in the way we have just described, without actually giving a meaning to the object $X^{\alpha}$. For example, a stable map from a pointed finite CW-complex $Z$ to $X^{\alpha}$ is an element of the group $\omega^{0}\left\{Z ; X^{\alpha}\right\}$, which can be defined exactly as above. To work with $X^{\alpha}$, one chooses a trivialization $\phi$ and takes $X^{\alpha}$ to be the stable space (or spectrum) $\Sigma^{-M} X^{\alpha_{0} \oplus \sigma}$. (Formally, $X^{\alpha}$ is the category of all such stable spaces, one for each $\phi$, with morphisms between any two the unique stable map giving the canonical identification. It is customary to regard such a category as a single object!)

Finally, we explain the isomorphism (4.1). If $\alpha_{1}$ is trivial, say $\alpha_{1}=X \times \mathbb{R}^{M}$, then it is easy to see that $\omega_{X}^{0}\left\{\alpha_{0}^{+} ; X \times\left(\mathbb{R}^{M}\right)^{+}\right\}$is (canonically isomorphic to) $\omega^{0}\left\{X^{\alpha_{0}} ;\left(\mathbb{R}^{M}\right)^{+}\right\}=$ 
$\tilde{\omega}^{M}\left(X^{\alpha_{0}}\right)$. See (9.10). In general, we fix a trivialization $\phi: \alpha_{1} \oplus \sigma \rightarrow \mathbb{R}^{M}$. The smash product over $X$ with the identity map on $\sigma^{+}$gives a suspension isomorphism

$$
\omega_{X}^{0}\left\{\alpha_{0}^{+} ; \alpha_{1}^{+}\right\} \rightarrow \omega_{X}^{0}\left\{\left(\alpha_{0} \oplus \sigma\right)^{+} ;\left(\alpha_{1} \oplus \sigma\right)^{+}\right\}
$$

Then $\phi$ induces an isomorphism to $\omega_{X}^{0}\left\{\left(\alpha_{0} \oplus \sigma\right)^{+} ; X \times\left(\mathbb{R}^{M}\right)^{+}\right\}=\tilde{\omega}^{M}\left(X^{\alpha_{0} \oplus \sigma}\right)$, which is $\tilde{\omega}^{0}\left(X^{\alpha}\right)$. It is straightforward to check that this isomorphism is independent of the choice of $\phi$.

\section{Duality}

We now describe the duality mentioned in the introduction. We continue with the notation of Sections 3 and 4 . In particular recall that the basepoints of $A, B, \ldots$ are written $a_{0}, b_{0}, \ldots$

Theorem 5.1. Suppose that $n \geqq 2 m+1$. Then the $C W$-complex $A$ of (3.4) may be chosen so that $A^{(n)}=A^{(2 n-2 m-1)}$ and so that this skeleton is $S$-dual to the stable Thom space $X^{-\zeta}$.

The proof is based on (4.6) together with the following facts which hold for a finite pointed CW-complex $P$ :

(a) Suppose that $1<r \leqq s$ and that $P$ is simply connected with $\tilde{H}_{j}(P ; \mathbb{Z})=0$ for $j<r$ and for $j>s$, and $\widetilde{H}^{s+1}(P ; \mathbb{Z})=0$. Then there is a finite pointed $C W$-complex $Q$, homotopy equivalent to $P$, with $Q^{(r-1)}=\left\{q_{0}\right\}$ and $\operatorname{dim} Q \leqq s$. (See [6], Ch. 8).

(b) Suppose that $r, s \geqq 0$, that $P^{(r+s-1)}=\left\{p_{0}\right\}$ and that $\operatorname{dim} P \leqq 2 r+s$. Then there is a finite pointed $\mathrm{CW}$-complex $Q$ with $Q^{(r-1)}=\left\{q_{0}\right\}$ and $\operatorname{dim} Q \leqq 2 r$, such that $P$ is homotopy equivalent to the $s$-fold suspension $\Sigma^{s} Q$. (See [1], Appendix).

Proof of (5.1). The result is trivially true for $m=0$. Assume that $m>0$.

Step 1. We choose a finite pointed CW-complex $D$ which is $S$-dual to $X^{-\zeta}$ and study its cell structure. We shall show that $D$ may be chosen so that $D^{(i)}=\left\{d_{0}\right\}$ for $0 \leqq i<n-m$ and $\operatorname{dim} D \leqq n$. For this step it is enough to assume $n \geqq 2 m$.

The special case when $X$ is a closed manifold is illuminating, since then $X^{-\zeta}$ is $S$-dual to the stable Thom space $X^{\zeta-\tau}$ where $\tau$ is the tangent bundle of $X$. The condition $n \geqq 2 m$ guarantees that $\zeta$ is equivalent to $\tau \oplus \eta$ for some $(n-m)$-plane bundle $\eta$ over $X$, and we may take $D=X^{\eta}$.

In general we use (a) and (b) above. Let $P$ be a finite pointed $C W$-complex which is $S$-dual to $\Sigma^{-N} X^{-\zeta}$ for some $N \geqq 0$. (In other words, if $\sigma \oplus \zeta=\mathbb{R}^{M}$ is trivial, $P$ is $S$-dual to $\Sigma^{-M-N} X^{\sigma}$.) Then $\tilde{H}_{j}(P ; \mathbb{Z})=0, \tilde{H}^{j}(P ; \mathbb{Z})=0$ unless $N+n-m \leqq j \leqq N+n$. By (a), there is a finite pointed $C W$-complex $Q$, also $S$-dual to $\Sigma^{-N} X^{-\zeta}$, with $Q^{(N+n-m-1)}=\left\{q_{0}\right\}$ and $\operatorname{dim} Q \leqq N+n$. Finally, since $n \geqq 2 m$ we see from (b) that $Q$ may be desuspended to a suitable $D: Q \simeq \Sigma^{N} D$.

Step 2. We next construct a duality map $j: D \rightarrow \Gamma \zeta^{+}$.

Let us choose a duality between $D$ and $X^{-\zeta ;}$ it will be given by stable maps $\lambda: D \wedge X^{-\zeta} \rightarrow S^{0}$ and $\mu: S^{0} \rightarrow D \wedge X^{-\zeta}$. (A good reference for duality theory is [4].) We regard $\lambda \in \tilde{\omega}^{0}\left(D \wedge X^{-\zeta}\right)$ as an element of $\omega^{0}\left(\left(D, d_{0}\right) \times X ; \zeta\right)$. By (4.6) $E:\left[D ; \Gamma \zeta^{+}\right] \rightarrow \omega^{0}\left(\left(D, d_{0}\right) \times X ;-\zeta\right)$ is surjective, since $m<n$. Choose $j$ such that $E(j)=\lambda$. 
Now for any finite pointed CW-complex $Z$ we have a commutative diagram

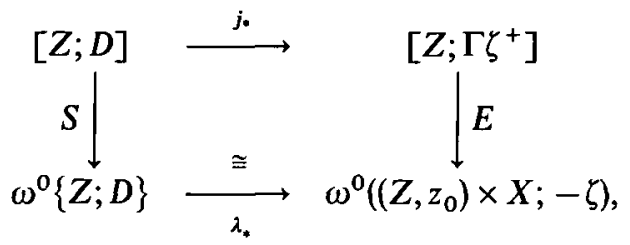

in which $S$ (rather than " $E$ ", which would be confusing here) is the ordinary stabilization map and $\lambda_{*}$ is the duality isomorphism. (A stable map $g: Z \rightarrow D$ gives rise to a stable map $\lambda \circ(g \wedge 1): Z \wedge X^{-\zeta} \rightarrow D \wedge X^{-\zeta} \rightarrow S^{0}$, hence to an element in $\tilde{\omega}^{0}\left(Z \wedge X^{-\zeta}\right)$, which (see (4.2)) is the same as $\omega^{0}\left(\left(Z, z_{0}\right) \times X ;-\zeta\right)$. The inverse for $\lambda_{*}$ is constructed using $\mu$.)

It is significant that the connection between function spaces and duality was already emphasized in the initial work of Spanier [14]. If $\zeta$ is the trivial bundle $\mathbb{R}^{n}$, then $j$ is a map $D \rightarrow M\left(X, S^{n}\right)$ to the space of maps from $X$ to $S^{n}$.

Step 3. $j$ induces an isomorphism of homotopy groups in an appropriate range.

Consider the diagram (5.2). By (4.6), $E$ is bijective for $\operatorname{dim} Z<2 n-m-1$. Since $D$ is $(n-m-1)$-connected, Freudenthal's suspension theorem shows that $S$, and hence $E \circ j_{*}$, is bijective for $\operatorname{dim} Z<2 n-2 m-1$, surjective for $\operatorname{dim} Z \leqq 2 n-2 m-1$. Hence $j_{*}$ satisfies the same conditions. Taking $Z$ to be a sphere we get the desired conditions on homotopy groups.

Step 4. If $n \geqq 2 m+1$, then $n \leqq 2 n-2 m-1$. We take $A^{(2 n-2 m-1)}=D$, mapped to $\left(\Gamma \zeta^{+}\right)^{0}$ by $j$, and continue the step-by-step construction of $A$ as in [16] (cf. (3.4) above). This completes the proof of (5.1).

The homotopy type of $\left(\Gamma \zeta^{+}\right)^{0}$ does not in general determine the homotopy type of the skeleton $A^{(i)}$. But if it happens that $A^{(i+1)}=A^{(i)}$, then this picks out a natural homotopy type for $A^{(i)}$. This occurs for $A^{(n)}$ when $n>2 m+1$, since then $2 n-2 m-1>n$, and so since $A^{(2 n-2 m-1)}=A^{(n)}$ we have certainly $A^{(n+1)}=A^{(n)}$.

Corollary 5.3. Suppose that $\zeta$ and $\eta$ are real $n$-plane bundles over $X$ with $n>2 m+1$, and that $\Gamma \zeta^{+}$and $\Gamma \eta^{+}$are (weakly) homotopy equivalent in dimensions $\leqq n$. Then the stable Thom spaces $X^{-\zeta}$ and $X^{-\eta}$ are homotopy equivalent.

Proof. Let $A$ be the CW-complex produced by Theorem 5.1 for $\Gamma \zeta^{+}$and let $B$ be the similar CW-complex corresponding to $\Gamma \eta^{+}$. Then under the current hypotheses, $A^{(n)}$ and $B^{(n)}$ are homotopy equivalent. Hence so are their $S$-duals $X^{-\zeta}$ and $X^{-\eta}$.

We note in particular:

Corollary 5.4. Suppose that $n>2 m+1$. Then $\Gamma^{+}$is homotopy equivalent to the space $M\left(X, S^{n}\right)$ of maps from $X$ to $S^{n}$ if and only if the sphere-bundle $\zeta^{+}$is fibre homotopy trivial. 


\section{Stabilization reformulated}

In this section we give two reformulations of stabilization, both giving less emphasis to the $\zeta^{+}$viewpoint than Section 4 . These will be used in Section 7 and Section 8 respectively.

As a preliminary, we recall some definitions (for example from Section 2 of [2]). The (stable cohomotopy) Euler class $\gamma(\xi)$ of $\xi$ is the class in $\omega^{0}(X ;-\xi)$, or equivalently in $\omega_{X}^{0}\left\{0^{+} ; \xi^{+}\right\}$, represented by the inclusion of $0^{+}$in $\xi^{+}$. In other words, $\gamma(\xi)$ is the stabilization of the zero-section of $\xi^{+}$. Let $s \in \Gamma(Y ; S \xi)$, where $Y$ is a subcomplex of $X$. The relative Euler class $\gamma(\xi, s)$ in $\omega^{\circ}(X, Y ;-\xi)$ is defined as follows. Let $D \xi$ be the unit disc bundle of $\xi$, and choose a section $\tilde{s}$ in $\Gamma(X ; D \xi)$ extending $s$. There is a standard (homotopy class of) bundle map $c: D \xi \rightarrow \xi^{+}$collapsing $S \xi$ fibrewise to $s_{+}$. The homotopy class of the composition $c \cdot \tilde{s}$ in $\Gamma\left(X, Y ; \xi^{+}\right)$is independent of the choice of $\tilde{s}$. Set $\gamma(\xi, s)=E(c \cdot \tilde{s})$. It clearly vanishes if $s$ extends to a section of $S \xi$ over $X$.

If sections $t_{0}, t_{1}$ of $S \xi$ over $X$ agree on $Y$, then the difference class $\delta\left(t_{0}, t_{1}\right)$ may be defined in $\omega^{-1}(X, Y ;-\xi)$ as follows. Let $p: X \times I \rightarrow X$ be the projection. Define a section $t$ of $S\left(p^{*} \xi\right)$ over $X \times \partial I \cup Y \times I$ to agree with $p^{*} t_{0}$ on $X \times\{0\}, p^{*} t_{1}$ on $X \times\{1\}$ and their common value on $Y \times I$. Then $\gamma\left(p^{*} \xi, t\right)$ lies in $\omega^{0}\left(X \times I, X \times \partial I \cup Y \times I ;-p^{*} \xi\right)$, and we define $\delta\left(t_{0}, t_{1}\right)$ to be its image in $\omega^{-1}(X, Y ;-\xi)$ under the suspension isomorphism. The difference class vanishes if $t_{0}$ and $t_{1}$ are homotopic. If $t_{0}, t_{1}, t_{2}$ are sections of $S \xi$ which agree on $Y$, then almost by definition of addition

$$
\delta\left(t_{0}, t_{2}\right)=\delta\left(t_{0}, t_{1}\right)+\delta\left(t_{1}, t_{2}\right)
$$

The next lemma expresses the stabilization map $E: \pi_{0} \Gamma\left(X, Y ; \zeta^{+}\right) \rightarrow \omega^{0}(X, Y ;-\zeta)$ in terms of difference classes. Recall that $s_{0}$ is a fixed section of $S \xi$ giving the splitting $\xi=\zeta \oplus \mathbb{R}$, and $S \xi=\zeta^{+}$. We may identify $\omega^{-1}(X, Y ;-\xi)$ with $\omega^{0}(X, Y ;-\zeta)$, from the definitions of these groups. Also, if $s$ is a section of $S \xi$ agreeing with $s_{0}$ on $Y$, then $s$ lies in $\Gamma\left(X, Y ; \zeta^{+}\right)$, so that the stabilization $E(s)$ of $s$ lies in $\omega^{0}(X, Y ;-\zeta)$.

Lemma 6.2. Under the above identification,

$$
E(s)=\delta\left(s, s_{0}\right)
$$

The proof is an exercise in relating reduced and unreduced fibrewise suspensions. We omit the details.

Under identifications similar to those above, the stabilization map of (4.5) becomes

$$
E:[Z ; \Gamma S \xi] \rightarrow \omega^{-1}\left(\left(Z, z_{0}\right) \times X ;-\xi\right)
$$

We want next to give a description of $E$ which does not involve the basepoint $s_{0}$ of $\Gamma S \xi$. This will be convenient when we come to discuss homology in Sections 8, 9 and in particular when we look at the action of symmetries of $S \xi$ which do not preserve the basepoint.

Let $C Z$ denote the (unreduced) cone $I \times Z /\{1\} \times Z$ on $Z$. Thus $Z \subseteq C Z$ as $\{0\} \times Z$. As usual the coboundary $\Delta: \omega^{-1}\left(\left(Z, z_{0}\right) \times X ;-\xi\right) \rightarrow \omega^{0}((C Z, Z) \times X ;-\xi)$ in the exact se- 
quence of the triple $\left(C Z, Z, z_{0}\right) \times X$ is an isomorphism, since $C Z$ is contractible to $z_{0}$. Given a pointed map $s: Z \rightarrow \Gamma S \xi$, its adjoint is a section, also called $s$, of (the pull-back of) $S \xi$ over $Z \times X$, agreeing with $s_{0}$ on $\left\{z_{0}\right\} \times X$. Thus on the one hand we have a difference class $\delta\left(s, s_{0}\right)$ in $\omega^{-1}\left(\left(Z, z_{0}\right) \times X ;-\xi\right)$ and on the other a relative Euler class $\gamma(\xi, s)$ in $\omega^{0}((C Z, Z) \times X ;-\xi)$.

\section{Lemma 6.3. With the above notation}

$$
\Delta \delta\left(s, s_{0}\right)=\gamma(\xi, s)
$$

The point of the lemma is that, by (6.2), the stabilization $E(s)$ is determined by $\delta\left(s, s_{0}\right)$, and so, by (6.3), stabilization may be viewed as a map from $[Z ; \Gamma S \xi]$ to $\omega^{0}((C Z, Z) \times X ;-\xi)$, avoiding any mention of $s_{0}$.

Proof of (6.3). It is convenient to split $\Delta$ into the composition:

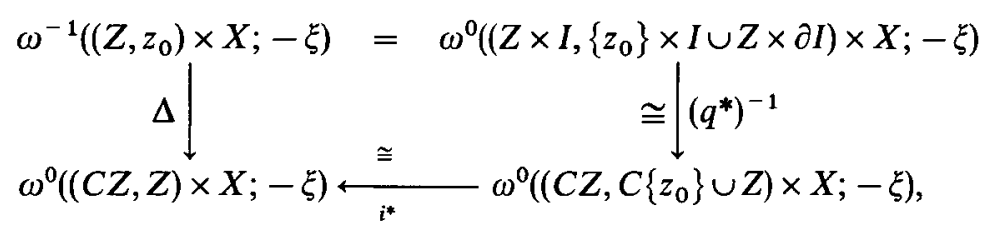

where $i$ is the inclusion and $q: Z \times I \rightarrow C Z$ is the collapsing map. We have a section $\bar{s}$ on $\left(C\left\{z_{0}\right\} \cup Z\right) \times X$ given by $s_{0}$ on $C\left\{z_{0}\right\} \times X$ and by $s$ on $Z \times X$. Then $q^{*} \gamma(\xi, \vec{s})=\delta\left(s, s_{0}\right)$ by definition of the difference class, and $i^{*} \gamma(\xi, \bar{s})=\gamma(\xi, s)$. Now (6.3) follows.

\section{Action of $\mathscr{G}$ on $\pi_{0} \Gamma S \xi$}

An element $g$ in $\mathscr{G}$ determines an element $\bar{g}$, say, in $K O^{-1}(X)$. (Explicitly, choose a trivialization $\phi: \xi \oplus \sigma \rightarrow \mathbb{R}^{N}$ for $N$ large. Then $g \oplus 1_{\sigma}$ gives rise to a map from $X$ to the orthogonal group $O(N)$ representing $\bar{g}$. It is easy to check that $\bar{g}$ is independent of the choice of $\sigma$ and $\phi$.) The $J$-homomorphism maps $K O^{-1}(X)$ to the group of units in the (unreduced) stable cohomotopy ring $\omega^{0}(X)$. The next lemma follows by unwinding definitions.

Lemma 7.1. With the above notation, $J(\bar{g})$ corresponds to the fibrewise one-point compactification $g^{+}: \xi^{+} \rightarrow \xi^{+}$under the natural isomorphism of $\omega^{0}(X)$ with $\omega^{0}(X ; \xi-\xi)=$ $\omega_{X}^{0}\left\{\xi^{+} ; \xi^{+}\right\}$.

The following obstruction-theoretic fact is also useful in studying the action of $\mathscr{G}$ on $\pi_{0} \Gamma S \xi$.

Lemma 7.2. Let $g \in \mathscr{G}, s \in \Gamma S \xi$. Then

$$
\delta\left(g s, s_{0}\right)=J(\bar{g}) \delta\left(s, s_{0}\right)+\delta\left(g s_{0}, s_{0}\right) .
$$


Proof. From (6.1), $\delta\left(g s, s_{0}\right)=\delta\left(g s, g s_{0}\right)+\delta\left(g s_{0}, s_{0}\right)$, and $\delta\left(g s, g s_{0}\right)=J(\bar{g}) \delta\left(s, s_{0}\right) \quad$ by naturality of $\delta$.

For an element $g \in \mathscr{G}$, let us define an affine linear map $\rho(g): \omega^{-1}(X ;-\xi) \rightarrow \omega^{-1}(X ;-\xi)$ by $\rho(g) x=J(\bar{g}) x+\delta\left(g s_{0}, s_{0}\right)$. Clearly $\rho(g)$ depends only on the component of $g$. It is not hard to check the cocycle condition: $\rho(g h)=\rho(g) \rho(h)$ for $g, h \in \mathscr{G}$. Now by (6.2) we can rephrase (7.2) in terms of $E: \pi_{0} \Gamma S \xi \rightarrow \omega^{0}(X ;-\xi)$ as:

$$
E(g \cdot s)=\rho(g) E(s)
$$

Before illustrating the use of these results, we describe $\rho(g)$ for certain symmetries $g$. The antipodal involution $T$ which acts as -1 in each fibre of $\xi$ is particularly interesting. Let $\eta$ denote the Hopf element which generates $\mathrm{KO}^{-1}(*)=\mathbb{Z} / 2$.

Proposition 7.4. (cf. [8]) Let $[\xi]$ be the class of $\xi$ in $K O^{\circ}(X)$. Then

(a) $\bar{T}=\eta \cdot[\xi]$ in $\mathrm{KO}^{-1}(X)$,

(b) $\delta\left(T s_{0}, s_{0}\right)=\gamma(\zeta)$

Proof. (a) If $\xi$ is the trivial bundle $\mathbb{R}$ over a point * then $\bar{T}=\eta$ essentially by definition of $\eta$. The general case quickly follows.

(b) By (6.2), $\delta\left(T s_{0}, s_{0}\right)=E\left(T s_{0}\right)$. But $T s_{0}$ is the zero-section of $\zeta^{+}$(in the usual identification of $S \xi$ with $\zeta^{+}$), so $E\left(T s_{0}\right)=\gamma(\zeta)$ by definition of $\gamma$ (see the beginning of Section 6).

Next, for any $t$ in $\Gamma S \xi$ let $R(t)$ be the (fibrewise) reflection of $\xi$ in the hyperplane orthogonal to $t$. Let $S(t)$ be the orientation-preserving element $R(t) R\left(s_{0}\right)$. Write $y=E(t)$ and $\varepsilon=J(\eta \cdot[\xi])$.

Proposition 7.5. With notation as above,

(a) $\rho\left(R\left(s_{0}\right) T\right) x=-\varepsilon x$,

(b) $\rho\left(R\left(s_{0}\right)\right) x=-x+\gamma(\zeta)$,

(c) $\rho(S(t)) x=x+(1+\varepsilon) y$,

for $x \in \omega^{-1}(X ;-\xi)$.

Proof. Since $\overline{R(t)}=-1$ and $R\left(s_{0}\right) s_{0}=T s_{0}$, (a) and (b) follow easily from (7.4). From (c) we use the identity $R(t) t=T t$. It follows that $\rho(R(t)) y=\rho(T) y=\varepsilon y+\gamma(\zeta)$. This enables us to compute $\rho(R(t))$ and then, using (b), $\rho(S(t))$.

Example 7.6. (cf. [5], [15]). Let $X$ be a closed connected $m$-manifold with $m$ odd, and let $\xi$ be an $(m+1)$-plane bundle over $X$ with $w_{1} \xi=w_{1} X$. Then $\pi_{0} \Gamma S \xi=\omega^{-1}(X ;-\xi) \cong \mathbb{Z}$, but we shall see that there are at most two orbits under the action of $\mathscr{G}$. For $\operatorname{dim} \zeta$ is odd, so $\gamma(\zeta)$ is a 2-torsion class and therefore $\gamma(\zeta)=0$ here. Also, $\varepsilon$ acts as +1 . The assertion follows from (7.5) (c).

In the case when $X$ is orientable and $\xi$ is trivial, this result was first proved in [5]. To see the connection, let $M$, denote the component of $M\left(X, S^{m}\right)$ consisting of all maps of degree $r$. Then (7.6) says that $\mathscr{G}$ provides strong equivalences (diffeomorphisms which are isometries) between $M_{r}$ and $M_{r+2 s}$ for any integers $r$ and $s$. 
Example 7.7. Let $X$ be a closed connected $m$-manifold with $m$ even and let $\xi$ be an $(m+1)$-plane bundle with $w_{1} \xi \neq w_{1} X$ and $w_{m} \xi \neq 0$. Then $\Gamma S \xi$ has two components and they are strongly equivalent by (7.5)(b).

\section{Homology of $\Gamma S \xi$}

In this section, continuing with the notation of previous sections, we deduce results about the homology of $\Gamma S \xi$ from the suspension theorem in Section 4. Since $\left(\Gamma \zeta^{+}\right)^{0}$ is of finite type by (3.4), the homology groups $\tilde{H}_{j}\left(\left(\Gamma \zeta^{+}\right)^{\circ}\right)$ are finitely generated. In a certain stable range they can be described in terms of the cohomology of $X$ (Corollary 8.3). This is deduced from a similar fact about stable homotopy and cohomotopy (Proposition 8.2). We then reformulate these results without reference to the basepoint $s_{0}$, preparing the way for the next section.

First we "stabilize" the map $E$ of (4.5).

Proposition 8.1 There exists a homomorphism $E_{*}$ making the following triangle commute:

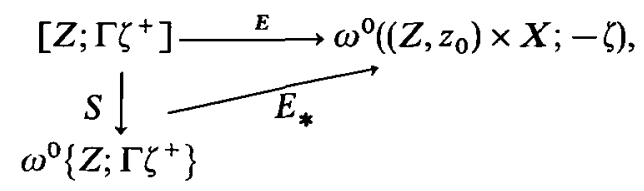

where $S$ (for clarity, instead of $E$ as elsewhere) is the ordinary stabilization map.

Proof. The proof is by universality. We shall therefore be dealing with "the stable cohomotopy of $\Gamma \zeta^{+}$. For our purposes, however, it is unnecessary to define this phrase: for " $\Gamma \zeta^{+"}$ one may simply read $A^{(N)}$ where $A$ is as in (3.4) and $N$ is sufficiently large.

Let 1 be the identity class in $\left[\Gamma \zeta^{+} ; \Gamma \zeta^{+}\right]$, and let $e$ be the universal class $E(1)$ in $\omega^{0}$ (“) $\left(\Gamma \zeta^{+}, s_{+}\right)$" $\left.\times X ;-\zeta\right)$. Since $E$ maps the class of $g: Z \rightarrow \Gamma \zeta^{+}$to $g^{*}(e)$, we may define $E_{*}$ by sending any stable map $g: Z \rightarrow \Gamma \zeta^{+}$to $g^{*}(e)$.

Now consider the case $Z=S^{j}$. The next proposition follows easily from (4.7), (8.1) and the usual Freudenthal suspension theorem applied to $S$.

Proposition 8.2. Suppose that $m<n$. Then $E_{*}: \tilde{\omega}_{j}\left(\Gamma \zeta^{+}\right) \rightarrow \omega^{-j}(X ;-\zeta)$ is an isomorphism for $j<2 n-2 m-1$, an epimorphism for $j \leqq 2 n-2 m-1$ (and indeed for $j<2 n-m$ ).

This gives rise to our first result on integral homology; it is just the homology analogue of (8.2). To state it, we use the local coefficient notation introduced in Section 4 , and we let $e$ denote also the Hurewicz image in $H^{0}\left(\left(\Gamma \zeta^{+}, s_{+}\right) \times X ;-\zeta\right)$ of the stable cohomotopy class $e$ above. (In cohomology there is no need for the inverted commas on $\Gamma \zeta^{+}$.) Taking the product with $e$ defines a map

$$
E_{*}: \tilde{H}_{j}\left(\Gamma \zeta^{+}\right) \rightarrow H^{-j}(X ;-\zeta)
$$

Corollary 8.3. The above $E_{*}$ is an isomorphism for $j<2 n-2 m-1$, an epimorphism for $j \leqq 2 n-2 m-1$. 
Proof. Let $P$ be a finite pointed CW-complex dual to $\Sigma^{-N} X^{-\zeta}$ for some $N \geqq 0$. Then $E_{*}$ is induced by a stable map $f: \Sigma^{N *} \Gamma \zeta^{+} " \rightarrow \mathrm{P}$. By (8.2), $f$ induces an isomorphism on the $j$ th stable homotopy group for $j<N+2 n-2 m-1$, an epimorphism for $j \leqq$ $N+2 n-2 m-1$. By the Whitehead theorem, it does the same on homology groups.

Remark 8.4. If $n \geqq 2 m+1$ we can apply the results of Section 5. (5.2) stabilizes to give

$$
\omega^{0}\{Z ; D\} \stackrel{j .}{\longrightarrow} \omega^{0}\left\{Z ; \Gamma \zeta^{+}\right\} \stackrel{E *}{\longrightarrow} \omega^{0}\{Z ; D\}
$$

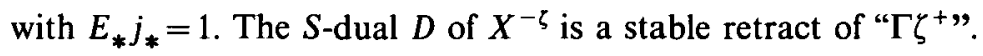

Next, for use in Section 9, we wish to rephrase (8.2) and (8.3) without mentioning $s_{0}$. Since $\tilde{\omega}_{j}\left(\Gamma \zeta^{+}\right)$may be identified with $\omega_{j}\left(\Gamma S \xi, s_{0}\right)$ and $\omega^{-j}(X ;-\zeta)$ with $\omega^{-(j+1)}(X ;-\xi)$, we first move to viewing $E_{*}$ as a map from $\omega_{j}\left(\Gamma S \xi, s_{0}\right)$ to $\omega^{-(j+1)}(X ;-\xi)$. Then as in Section 6 we can "get rid of" $s_{0}$ by using the boundary isomorphism $\partial: \omega_{j+1}(C \Gamma S \xi, \Gamma S \xi) \rightarrow \omega_{j}\left(\Gamma S \xi, s_{0}\right)$. In order to get the desired translation of $(8.2)$ we need a lemma.

Lemma 8.5. The composition

$$
E_{*} \partial: \omega_{j+1}(C \Gamma S \xi, \Gamma S \xi) \rightarrow \omega^{-(j+1)}(X ;-\xi)
$$

is given by multiplication by the relative Euler class $\gamma(\xi, 1)$ in $\omega^{0}$ (“( $(C \Gamma S \xi, \Gamma S \xi)$ ” $\left.\times X ;-\xi\right)$, where 1 is the canonical section of (the pull-back of) $S \xi$ over $\Gamma S \xi \times X$ given by the identity map of $\Gamma S \xi$.

Proof. This follows from (6.3) applied with $Z=$ " $\Gamma S \xi$ ".

Now (8.2) and (8.3) immediately give corresponding statements in which $E_{*}$ is replaced by multiplication by the Euler class $\gamma(\xi, 1)$ in stable cohomotopy or in integral cohomology. We record these as:

Corollary 8.6. Let $m<n$. Then

$$
\gamma(\xi, 1) \cdot: \omega_{j+1}(C \Gamma S \xi, \Gamma S \xi) \rightarrow \omega^{-(j+1)}(X ;-\xi)
$$

is an isomorphism for $j<2 n-2 m-1$, an epimorphism for $j \leqq 2 n-2 m-1$.

Corollary 8.7. Let $m<n$. Then

$$
\gamma(\xi, 1) \cdot H_{j+1}(C \Gamma S \xi, \Gamma S \xi) \rightarrow H^{-(j+1)}(X ;-\xi)
$$

is an isomorphism for $j<2 n-2 m-1$, an epimorphism for $j \leqq 2 n-2 m-1$. 
Since $\partial: H_{j+1}(C \Gamma S \xi, \Gamma S \xi) \rightarrow H_{j}(\Gamma S \xi)$ is an isomorphism, (8.7) shows that we can express $H_{j}(\Gamma S \xi)$ as $H^{-(j+1)}(X ;-\xi)$ provided $0<j<2 n-2 m-1$. (This of course follows from (5.1) in the special case $n \geqq 2 m+1$.)

Remark 8.8. A symmetry $g$ in $\mathscr{G}$ acts on $H^{-(j+1)}(X ;-\xi)$ as multiplication by the degree of $g$ in $H^{0}(X)$. In particular if $g$ is orientation-preserving (that is, of degree +1 ) then it acts trivially on $H_{j}(\Gamma S \xi)$ for $j<2 n-2 m-1$, since everything involved in the isomorphism of $H_{j}(\Gamma S \xi)$ with $H^{-(j+1)}(X ;-\xi)$ is natural with respect to $g$.

\section{A "Gysin sequence"}

Our immediate goal is a bundle version of (8.7), from which we shall deduce a kind of Gysin sequence for a bundle with fibre $\Gamma S \xi$ (Corollary 9.4). We conclude the section with some applications, including an alternative proof of Theorem 2.13 in [3].

Suppose that $p: E \rightarrow B$ is a locally trivial bundle over a finite CW-complex $B$, and that for each point $b$ in $B$ the fibre $E_{b}$ over $b$ is homeomorphic to $X$. Suppose that $\rho$ is a real $(n+1)$-plane bundle over $E$ equipped with a Riemannian metric. Write $\rho_{b}=\rho \mid E_{b}$. We assume that $m<n$, so that the space of sections $\Gamma S \rho_{b}$, or $\Gamma_{b}$ for short, is non-empty and connected. The $\Gamma_{b}$ can be assembled as the fibres of a locally trivial bundle $\pi: \Gamma \rightarrow B$. Let $C_{B} \pi: C_{B} \Gamma \rightarrow B$ be the cone over $B$ of $\pi$. Thus $\Gamma \subseteq C_{B} \Gamma$ and the pointed space $C_{B} \Gamma / \Gamma$ is the mapping cone of $\pi$. This gives an exact sequence in homology (and likewise in stable homotopy):

$$
\ldots H_{j+1}(\Gamma) \stackrel{\pi_{*}}{\longrightarrow} H_{j+1}(B) \stackrel{k}{\longrightarrow} H_{j+1}\left(C_{B} \Gamma, \Gamma\right) \stackrel{\partial}{\longrightarrow} H_{j}(\Gamma) \rightarrow \ldots
$$

Using (8.7) we shall compute $H_{j+1}\left(C_{B} \Gamma, \Gamma\right)$ for $j<2 n-2 m-1$ and thus obtain an exact sequence which can be applied to the calculation of the groups $H_{f}(\Gamma)$ in this range.

Let $S_{B} \Gamma$ be the fibre suspension of $\Gamma$; its fibre over a point $b$ of $B$ is $S \Gamma_{b}=C \Gamma_{b} / \Gamma_{b}$. Let $R$ be the stable bundle over $B$ with fibre over $b$ the stable Thom space $R_{b}=E_{b}^{\left(-\rho_{b}\right)}$; then $R / B$ is the stable Thom space $E^{-\rho}$. (To work with genuine spaces, one chooses a trivialization of $\rho \oplus \sigma$ for some vector bundle $\sigma$ over $E$ and considers the bundle with fibre at $b$ the Thom space of $\sigma \mid E_{b}$.)

The computation of $H_{j+1}\left(C_{B} \Gamma, \Gamma\right)$ is an exercise in homotopy theory over $B$ and in essence it is straightforward. Suppose that $\Delta_{b}$ is a (stable) dual of $R_{b}$. Then $\gamma\left(\rho_{b}, 1\right)$ in $\omega^{0}$ ("' $\left(C \Gamma_{b}, \Gamma_{b}\right)$ " $\left.\times E_{b} ;-\rho_{b}\right)$ can be regarded as a stable map $\theta_{b}:$ : $S \Gamma_{b} " \rightarrow \Delta_{b}$, which, according to (8.7), induces an isomorphism $\tilde{H}_{j+1}\left(S \Gamma_{b}\right) \rightarrow \tilde{H}_{j+1}\left(\Delta_{b}\right)$ for $j<2 n-2 m-1$, an epimorphism for $j \leqq 2 n-2 m-1$. Now suppose that we could assemble the spaces $\Delta_{b}, b \in B$, into a locally trivial (stable) bundle over $B, S$-dual over $B$ to the bundle $R$, and the $\theta_{b}$ into a stable map $\theta:$ : $S_{B} \Gamma$ " $\rightarrow \Delta$ over $B$. Then $\theta_{*}: \tilde{H}_{j+1}\left(S_{B} \Gamma / B\right) \rightarrow \tilde{H}_{j+1}(\Delta / B)$ would be an isomorphism in the same range $j<2 n-2 m-1$, an epimorphism for $j \leqq 2 n-2 m-1$. This is the substance of the computation. However, we do not wish to discuss general duality theory over $B$ and shall, therefore, proceed slightly differently.

For simplicity we assume that $B$ is a closed manifold with tangent bundle $\tau$. (No doubt the theory can be carried through without this restriction, but we have not done so.) Here is the result. In the statement $D$ denotes the canonical duality isomorphism. 
Proposition 9.2. There is a natural map $\theta$ making the following diagram commute:

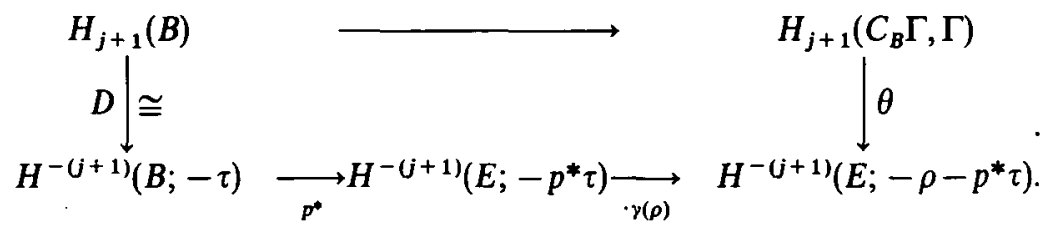

Moreover, $\theta$ is an isomorphism for $j<2 n-2 m-1$, an epimorphism for $j \leqq 2 n-2 m-1$.

Note that if $B$ is a single point then (9.2) reduces to (8.7). We shall in fact prove a stable homotopy version of the proposition (from which the isomorphisms in the homology version follow by the usual Whitehead argument). For this we need the following result from fibre homotopy theory. It is proved in the appendix.

Lemma 9.3. Let $Z$ be a finite pointed $C W$-complex, $p: Q \rightarrow B$ a locally trivial bundle of pointed finite $C W$-complexes. Then there is an isomorphism

$$
\alpha: \omega^{*}\{Z ; Q / B\} \rightarrow \omega_{B}^{*}\left\{B \times Z ; \tau^{+} \wedge_{B} Q\right\},
$$

which is natural in $Z$ and $Q$ and which reduces when $Z=S^{0}$ and $Q=B \times S^{0}$ to the canonical duality isomorphism of

$$
\omega_{-*}(B)=\omega^{*}\left\{S^{0} ;\left(B \times S^{0}\right) / B\right\} \text { with } \omega^{*}(B ;-\tau)=\omega_{B}^{*}\left\{B \times S^{0} ; \tau^{+}\right\} .
$$

Proof of the stable homotopy version of (9.2). By (9.3) we can identify $\omega_{-*}\left(C_{B} \Gamma, \Gamma\right)=$ $\omega^{*}\left\{S^{0} ; S_{B} \Gamma / B\right\}$ with $\omega_{B}^{*}\left\{B \times S^{0} ; \tau^{+} \wedge_{B} S_{B} \Gamma\right\}$. Note also, from the definitions, that $\omega^{*}\left(E ;-\rho-p^{*} \tau\right)=\omega_{B}^{*}\left\{R ; \tau^{+}\right\}$.

Now consider the relative Euler class $\gamma(\rho, 1)$ in

$$
\left.\omega^{0} \text { (“" }\left(C_{B} \Gamma, \Gamma\right) " \times{ }_{B} E ;-\rho\right)=\omega_{B}^{0}\left\{“ S_{B} \Gamma " \wedge_{B} R ; 0^{+}\right\}
$$

Multiplication by $\gamma(\rho, 1)$ gives a homomorphism $\theta$ :

$$
\omega_{j+1}\left(C_{B} \Gamma, \Gamma\right) \rightarrow \omega^{-(j+1)}\left(E ;-\rho-p^{*} \tau\right)
$$

or

$$
\omega_{B}^{-(j+1)}\left\{0^{+} ; \tau^{+} \wedge_{B} S_{B} \Gamma\right\} \rightarrow \omega_{B}^{-(j+1)}\left\{R ; \tau^{+}\right\}
$$

according to our point of view.

At the fibre level, (8.6) tells us (in view of the identifications noted above) that $\theta_{b}: \omega^{-(j+1)}\left\{0^{+} ; \tau_{b}^{+} \wedge S \Gamma_{b}\right\} \rightarrow \omega^{-(j+1)}\left\{R_{b} ; \tau_{b}^{+}\right\}$is an isomorphism for $j<2 n-2 m-1+\operatorname{dim} B$, an epimorphism for $j \leqq 2 n-2 m-1+\operatorname{dim} B$. It follows that $\theta$ is an isomorphism or epimorphism in the range claimed. (If $B$ has the structure of a finite $\mathrm{CW}$-complex then it is easy to argue by induction over the cells, using the exact sequence relating stable homotopy over $B$, over a subcomplex $A$ and over $(B, A)$.) 
Finally, we see from (9.3) that $k: \omega_{j+1}(B) \rightarrow \omega_{j+1}\left(C_{B} \Gamma, \Gamma\right)$ corresponds under $\alpha$ to the map from $\omega_{B}^{-(j+1)}\left\{0^{+} ; \tau^{+}\right\}$to $\omega_{B}^{-(j+1)}\left\{0^{+} ; \tau^{+} \wedge_{B} S_{B} \Gamma\right\}$ induced by the inclusion of $B$ in $S_{B} \Gamma$ as the vertex of the cone. Then composition with $\theta$ gives multiplication by the Euler class $\gamma(\rho)$. This completes the proof.

Bundles of the type we have been discussing arise in the following way. Ley $\mathscr{H} \subseteq \mathscr{G}$ be the subgroup of orientation-preserving symmetries of $\xi$. Suppose that $P$ is the total space of a (locally trivial) principal $\mathscr{H}$-bundle over $B$, and let $E=B \times X$, with $p: B \times X \rightarrow B$ the projection, and $\rho=P \times \not x \xi$. (See Example 9.7 below for a specific example of the type we have in mind.) Write $q: B \times X \rightarrow X$ for the projection onto $X$. Since $\mathscr{H}$ is orientation-preserving the orientation bundle of $\rho$ is identified with that of $q^{*} \xi$, and we have a Thom class $u$, say, in $H^{\circ}\left(B \times X ; \rho-q^{*} \xi\right)$.

In applying (9.2), it is convenient to use the following notation in cohomology, by analogy with stable cohomotopy: for finite pointed $\mathrm{CW}$-complexes $Z_{0}, Z_{1}$ we write $H^{*}\left\{Z_{0} ; Z_{1}\right\}$ for the group of chain homotopy classes of chain maps from the reduced chain complex $\tilde{C}_{*}\left(Z_{0}\right)$ to $\tilde{C}_{*}\left(Z_{1}\right)$. (Thus, $H^{j}\left\{Z_{0} ; S^{0}\right\}$ is cohomology $\tilde{H}^{j}\left(Z_{0}\right)$ and $H^{j}\left\{S^{0} ; Z_{1}\right\}$ is homology $\tilde{H}_{-}\left(Z_{1}\right) . H^{0}\left\{Z_{0} ; Z_{1}\right\}$ should be thought of as the morphisms from $Z_{0}$ to $Z_{1}$ in the "homology category". In the context of general duality theory described in [4], it is transparent that $H^{*}\left\{Z_{0} ; Z_{1}\right\}$ can be identified with $\tilde{H}^{*}\left(Z_{0} \wedge D\left(Z_{1}\right)\right)$, where $D\left(Z_{1}\right)$ is an $S$-dual of $Z_{1}$. We shall use this identification in the proof of (9.4) below. For the computation of $H^{*}\left\{Z_{0} ; Z_{1}\right\}$ one has a (split) short exact sequences:

$$
0 \rightarrow \operatorname{Ext}\left(\tilde{H}_{*}\left(Z_{0}\right), \tilde{H}_{*}\left(Z_{1}\right)\right) \rightarrow H^{*}\left\{Z_{0} ; Z_{1}\right\} \rightarrow \operatorname{Hom}\left(\tilde{H}_{*}\left(Z_{0}\right), \tilde{H}_{*}\left(Z_{1}\right)\right) \rightarrow 0
$$

or its analogue involving the cohomology groups of $Z_{0}$ and $Z_{1}$.)

Now let $e$ be the Euler class $\gamma(\rho) \cdot u$ in $H^{0}\left(B \times X ;-p^{*} \xi\right)$. We write $B^{+}$for the disjoint union of $B$ with a basepoint.

Corollary 9.4. With notation as above, there is an exact sequence:

$$
\ldots \rightarrow H_{j+1}(B) \stackrel{e \cdot}{\longrightarrow} H^{-(j+1)}\left\{X^{-\xi} ; B^{+}\right\} \rightarrow H_{j}\left(P \times_{\mathscr{H}} \Gamma S \xi\right) \stackrel{p_{*}}{\longrightarrow} H_{j}(B) \rightarrow \ldots
$$

for $j<2 n-2 m-1$.

Proof. By standard considerations it is enough to assume that $B$ is a closed manifold. (In general, a finite complex $B$ is a retract of a closed manifold.) The result now follows from (9.1) and (9.2). For by $(9.2) H_{*}\left(C_{B} \Gamma, \Gamma\right)$ is isomorphic via $\theta$ to $H^{-*}\left(B \times X ;-\rho-p^{*} \tau\right)$, which in turn is identified with $H^{-*}\left(B \times X ;-p^{*} \tau-q^{*} \xi\right)$ via the Thom isomorphism (multiplication by $u$ ). Now this last group is, by definition, $\tilde{H}^{-*}\left(B^{-\tau} \wedge X^{-\xi}\right)$, which is identified by the canonical duality between $B^{-\tau}$ and $B^{+}$with $H^{-*}\left\{X^{-\xi} ; B^{+}\right\}$. The map $k$ in (9.1) passes to multiplication by $e$ when $H^{-(j+1)}\left\{X^{-\xi} ; B^{+}\right\}$ is substituted for $H_{j+1}\left(C_{B} \Gamma, \Gamma\right)$. This establishes (9.4).

Remark 9.5. In (9.4) we can allow $B$ to be an arbitrary CW-complex. For then it is a direct limit of its finite subcomplexes and (9.4) is compatible with the direct limit 
process. We can also replace $\mathscr{H}$ by an arbitrary subgroup $\mathscr{H}^{\prime} \subseteq \mathscr{H}$. For if $P^{\prime} \rightarrow B$ is a principal $\mathscr{H}^{\prime}$-bundle we can extend it to $P=P^{\prime} \times \mathscr{H}^{\prime} \mathscr{H} \rightarrow B$ and then $P^{\prime} \times \mathscr{H}^{\prime} \Gamma S \xi=$ $P \times \not{H} \Gamma S \xi$.

Remark 9.6. Using homology with $\mathbb{F}_{2}$-coefficients, we get a result like (9.4)-(9.5) with $\mathscr{H}$ replaced by $\mathscr{G}$. Notice that for field coefficients $H^{*}\left\{Z_{0} ; Z_{1}\right\}$ is just $\operatorname{Hom}\left(\widetilde{H}_{*}\left(Z_{0}\right), \tilde{H}_{*}\left(Z_{1}\right)\right)$. So $H^{-(j+1)}\left\{X^{-\xi} ; B^{+}\right\}$is easy to compute in this case.

Example 9.7. Suppose that $X$ is a connected oriented closed even-dimensional manifold, of dimension $m=2 r$, and that $\xi$ is a complex $s$-plane bundle (with Hermitian metric) over $X$ with $n=2 s-1$ and $r<s$. We take $\mathscr{H}=S^{1}$ (as in (9.5)) acting on $\xi$ by complex scalar multiplication and $P \rightarrow B$ the universal $S^{1}$-bundle $S^{\infty} \rightarrow \mathbb{C} P^{\infty}$. Then $\rho$, in the proof of (9.4), is $H \otimes_{c} \xi$, where $H$ is the Hopf line bundle over $\mathbb{C} P^{\infty}$. Because $S^{1}$ acts freely on $\Gamma S \xi$, we have an isomorphism: $H_{*}\left(S^{\infty} \times{ }_{S_{1}} \Gamma S \xi\right) \rightarrow H_{*}\left(\Gamma S \xi / S^{1}\right)$. A routine calculation using the exact sequence of (9.4) now yields:

$$
H_{n-m}\left(\Gamma S \xi / S^{1} ; \mathbb{Z}\right)=\mathbb{Z} / c_{r} \xi[X]
$$

where $c_{r} \xi$ is the $r$ th Chern class and $[X]$ the fundamental homology class.

From Section 8 of [3] (especially Lemma 8.2) it follows that if $H^{1}(X ; \mathbb{Z})=0$ then $\Gamma S \xi / S^{1}$ is homotopy equivalent to a certain space $N \xi$ of sections of the projective bundle $\mathbb{C} P \xi$. The above calculation therefore gives an alternative proof of Theorem 2.13 of [3] (for $m<n$ ). Other results first proved by Møller in [11], [12], [13] can be obtained similarly from (9.4).

Example 9.8. (cf. Møller [12] (3.4).) Suppose that $X$ is a connected closed $m$-manifold and $\xi$ a real $(n+1)$-plane bundle with $m \leqq n$. We may take $\mathscr{G}=\mathbb{Z} / 2$ in (9.6), acting on $\xi$ as multiplication by $\{ \pm 1\}$, and $P \rightarrow B$ the universal $\mathbb{Z} / 2$-bundle. Again the action on $\Gamma S \xi$ is free, and we obtain:

$$
H_{n-m}\left(\Gamma S \xi /(\mathbb{Z} / 2) ; \mathbb{F}_{2}\right)=\mathbb{F}_{2} \oplus\left(\mathbb{F}_{2} / w_{m} \xi[X]\right),
$$

where $w_{m} \xi$ is the $m$ th Stiefel-Whitney class.

Remark 9.9. In this and the preceding section we have dealt only with stable homotopy and classical cohomology. There are corresponding results for any connective (multiplicative) homology theory. For example, suppose that $\xi$ is a complex (Hermitian) bundle, $\mathscr{H}$ the unitary subgroup of $\mathscr{G}$. Then we can replace $H$ in (9.4) by connective complex $K$-theory.

\section{Appendix: stable homotopy over a base space}

We shall be doing homotopy theory over a fixed finite CW-complex $B$. Let $p: Q \rightarrow B$ be a locally trivial bundle of pointed finite $\mathrm{CW}$-complexes. Then $Q$ is a sectioned space over $B$ and we shall sometimes regard the section as an inclusion $B \subseteq Q$. Let $Z$ be a pointed finite $C W$-complex, and consider the product bundle $B \times Z \rightarrow B$. There is an 
evident correspondence between fibrewise pointed maps $Q \rightarrow B \times Z$ over $B$ and pointed maps $Q / B \rightarrow Z$. This observation leads easily to the well known lemma:

Lemma 9.10. There is a canonical isomorphism between $\omega_{B}^{*}\{Q ; B \times Z\}$ and $\omega^{*}\{Q / B ; Z\}$.

In particular, $\omega_{B}^{*}\left\{Q ; B \times S^{0}\right\}$, which one might call the "stable cohomotopy of $Q$ over $B$ ", is equal to $\tilde{\omega}^{*}(Q / B)$, the stable cohomotopy of $Q / B$. It is natural to ask for a similar interpretation of the "stable homotopy of $Q$ over $B$ ", $\omega_{B}^{*}\left\{B \times S^{0} ; Q\right\}$. The purpose of this appendix is to give such an interpretation, at least if, as we now assume, $B$ is a closed smooth manifold. We write $\tau$ for the tangent bundle of $B$.

Lemma 9.11. With the above notation, there is a natural isomorphism

$$
\alpha: \omega^{*}\left\{Z ;\left((-\tau)^{+} \wedge_{B} Q\right) / B\right\} \rightarrow \omega_{B}^{*}\{B \times Z ; Q\} .
$$

If we fix a trivialization $\tau \oplus v \rightarrow \mathbb{R}^{N}$, for some normal bundle $v$, then we can express the group on the left in more concrete terms as $\omega^{*-N}\left\{Z ;\left(v^{+} \wedge_{B} Q\right) / B\right\}$. The equivalence $\alpha$ here is essentially the same as that in (9.3); we can go from (9.3) to (9.11) by substituting $v^{+} \wedge_{B} Q$ for $Q$. The remainder of this appendix is concerned with the proof of (9.3). Although we know of no reference, the result is more or less standard. We therefore only indicate the main steps of the proof here.

We need to recall the general construction of the Umkehr map. Suppose first that $f: X \rightarrow Y$ is a smooth map between closed manifolds $X$ and $Y$ with tangent bundles $\tau X, \tau Y$. Let $R \rightarrow Y$ be a locally trivial bundle of pointed finite CW-complexes over $Y$, and $f^{*} R \rightarrow X$ its pull-back over $X$. Then there is a natural stable map (depending only on the homotopy class of $f$ )

$$
f^{!}:\left((-\tau Y)^{+} \wedge_{Y} R\right) / Y \rightarrow\left((-\tau X)^{+} \wedge_{x} f^{*} R\right) / X
$$

which we shall call the Umkehr map. (It has many names.) The construction is easiest to describe if $f$ is an embedding of a submanifold $X \subseteq Y$ with normal bundle $v$. We write $D v$ for the unit disc bundle and identify its interior $D v-S v$ with $v$ in the usual way. Choose a tubular neighbourhood $D v \subseteq Y$ of $X$ and identify the restriction $R \mid D v$ with the pull-back $(D v) \times{ }_{X}(R \mid X)$ by an isomorphism restricting to the identity on $X$. Now we generalize the standard Pontrjagin-Thom construction. Collapsing $(R \mid(Y-v)) \cup Y$ in $R$ and $\left((S v) \times{ }_{X}(R \mid X)\right) \cup D v$ in $(D v) \times{ }_{X}(R \mid X)$ to points, we obtain a map

$$
f^{\prime}: R / Y \rightarrow\left(v^{+} \wedge_{X} f^{*} R\right) / X
$$

Substituting $(-\tau Y)^{+}{ }_{{ }_{Y}} R$ for $R$, we obtain the more symmetrical form (9.12). In general, if $f$ is not an embedding one chooses an embedding $i: X \rightarrow V$ of $X$ in some Euclidean space $V$ and applies the above Pontrjagin-Thom construction to $(f, i): X \rightarrow Y \times V$.

The construction carries through, virtually unchanged, over $B$. Let $p: E \rightarrow B, q: F \rightarrow B$ be locally trivial smooth fibre bundles over $B$ with fibres closed manifolds. Write $\tau(p), \tau(q)$ for the bundles of tangents along the fibres. (In fact we do not need a differentiable 
structure on $B$; it is enough that $E, F$ be manifolds over $B$.) Let $f$ now be a smooth map $E \rightarrow F$ over $B$ and $R \rightarrow F$ a locally trivial bundle of pointed finite $C W$-complexes over $F$. Then we have a stable map over $B$

$$
f^{\prime}:\left((-\tau(q))^{+} \wedge_{F} R\right) /{ }_{B} F \rightarrow\left((-\tau(p))^{+} \wedge_{E} f^{*} R\right) /{ }_{B} E,
$$

where $/_{B}$ is the quotient over $B$ (see [9]) collapsing a subspace to a point in each fibre.

Sketch proof of (9.3). Recall first how the canonical duality between $B^{+}$(the onepoint-compactification of $B$, with basepoint at $\infty$ ) and $B^{-\tau}$ is defined. It will be convenient to denote a point by 0 , so that $0^{+}$is the pointed space $S^{0}$. We require two structure maps $\lambda: B^{+} \wedge B^{-\tau} \rightarrow 0^{+}, \mu: 0^{+} \rightarrow B^{+} \wedge B^{-\tau}$ satisfying certain identities. Let $\Delta: B \rightarrow B \times B$ be the diagonal and $\pi: B \rightarrow 0$ the constant map. We write $\pi_{1}, \pi_{2}: B \times B \rightarrow B$ for the projections onto the first and second factor, so that $B^{+} \wedge B^{-\tau}=(B \times B)^{-\pi_{2}^{*} \tau}$.

Then $\lambda$ is the composition

$$
(B \times B)^{-\pi_{2}^{*} \tau} \stackrel{\Delta^{!}}{\longrightarrow} B^{+} \stackrel{\pi}{\longrightarrow} 0^{+}
$$

and dually $\mu$ is

$$
0^{+} \stackrel{\pi^{!}}{\longrightarrow} B^{-\tau} \stackrel{\Delta}{\longrightarrow}(B \times B)^{-\pi_{2}^{*} \tau}
$$

For $\Delta^{!}$we take $R=\pi_{1}{ }^{*} \tau^{+}$in (9.12). We identify $\Delta^{*} \pi_{1}{ }^{*} \tau$ and $\Delta^{*} \pi_{2}{ }^{*} \tau$ with $\tau$. One verifies that $\lambda, \mu$ are duality maps by an essentially formal argument using the properties of the Umkehr construction.

We shall define the transformation $\alpha$ in a rather similar way. It is enough to consider the case $Z=Q / B$ and define $\alpha(1)$, where 1 in $\omega^{0}\{Q / B ; Q / B\}$ is the identity map. (For then, in general, if $x$ is in $\omega^{0}\{Z ; Q / B\}$ we can set $\alpha(x)=(1 \times x)^{*} \alpha(1)$.) We use the notation of (9.14). Take $1: B \rightarrow B$ for $p$ and $\pi_{1}: B \times B \rightarrow B$ for $q$. Then $f$ will be $\Delta$ and $R$ will be $\pi_{2}^{*}\left(\tau^{+} \wedge{ }_{B} Q\right)$. Thus:

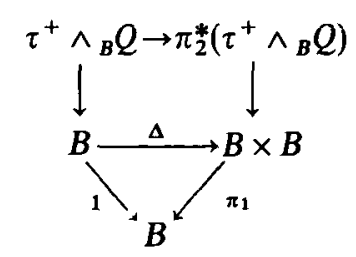

From (9.14) we get $\Delta^{\prime} \in \omega_{B}^{0}\left\{\mathrm{~B} \times(Q / B) ; \tau^{+} \wedge_{B} Q\right\}$, because $\tau(p)=0$ and $\tau(q)=\pi_{2}{ }^{*} \tau$. We define $\alpha(1)$ to be the Umkehr map $\Delta$ !.

To show that $\alpha$ is an isomorphism we construct an inverse $\beta$. It is convenient to identify $\omega_{B}^{*}\left\{(-\tau)^{+} \wedge_{B}(B \times Z) ; Q ;\right.$ with $\omega_{B}^{*}\left\{B \times Z ; \tau^{+} \wedge_{B} Q\right\}$ by the fibrewise suspension 
(product with the identity map on $\tau^{+}$) and define $\beta$ as a composition:

$$
\omega_{B}^{*}\left\{(-\tau)^{+} \wedge_{B}(B \times Z) ; Q\right\} \stackrel{\mathcal{c}}{\longrightarrow} \omega^{*}\left\{B^{-\tau} \wedge Z ; Q / B\right\} \stackrel{\pi_{\bullet}}{\longrightarrow} \omega^{*}\{Z ; Q / B\}
$$

Here $c$ collapses the section $B$ to a point, and $\pi_{*}$ is composition with $\pi^{\prime}: 0^{+} \rightarrow B^{-\tau}$.

The proof is completed by what is again a rather formal verification that $\alpha$ and $\beta$ are inverse to one another.

Remark 9.18. There is one case in which one can give a rather simpler proof, namely when $Q \rightarrow B$ is the fibrewise one-point compactification of a locally trivial smooth fibre bundle $p: E \rightarrow B$ with closed fibre. Then one can use duality over $B$ to identify $\omega_{B}^{*}\left\{B \times Z ; \tau^{+} \wedge_{B} Q\right\}$ with $\omega_{B}^{*}\left\{Z \times\left((-\tau E)^{+} /{ }_{B} E\right) ; 0^{+}\right\}$, since $\tau E$ is the direct sum $\tau B \oplus \tau(p)$. This group is, by $(9.10), \tilde{\omega}^{*}\left(Z \wedge E^{-\tau E}\right)$, which by ordinary duality is $\omega^{*}\left\{Z ; E^{+}\right\}$. And finally, $E^{+}$is $Q / B$.

\section{REFERENCES}

1. M. G. Barratr, Homotopy ringoids and homotopy groups, Quart. J. of Math. (Oxford) (2) 5 (1954), 271-290.

2. M. C. CRABb, $\mathbb{Z} / 2$-Homotopy Theory (Cambridge University Press, 1980).

3. M. C. Crabb and W. A. Sutherland, Function spaces and Hurwitz-Radon numbers, Math. Scand. 55 (1984), 67-90.

4. A. Dold and D. Puppe, Duality, trace and transfer, in Proc. Int. Conf. on Geometric Topology, 81-102 (ed. K. Borsuk and A. Kirkor, PWN, Warsaw, 1980).

5. V. L. Hansen, On spaces of maps of $n$-manifolds into the $n$-sphere, Trans. Amer. Math. Soc. 265 (1981), 273-281.

6. P. Hilton, Homotopy theory and duality (Nelson, London, 1967).

7. P. Hilton, G. Mislin and J. Roitberg, Localization of nilpotent spaces (North-Holland, Amsterdam, 1975).

8. I. M. James, On sphere-bundles I, Bull. Amer. Math. Soc. 75 (1969), 617-621.

9. I. M. JAMES, General Topology and Homotopy Theory (Springer, New York, 1984).

10. J. MiLNOR. On spaces having the homotopy type of CW-complexes. Trans. Amer. Math. Soc. 40 (1959), 272-280.

11. J. M. Møller, On spaces of maps between complex projective spaces, Proc. Amer. Math. Soc. 91 (1984), 471-476.

12. J. M. Møller, On the cohomology of spaces of sections of real projective bundles (Preprint Series 1983 No. 12, Kobenhavns Universitet, Matematisk Institut).

13. J. M. Møller, On the homology of spaces of sections of complex projective bundles, Pacific J. Math. 116 (1985), 143-154. 
14. E. H. Spanier, Function spaces and duality, Ann. of Math. (2) 70 (1959), 338-378.

15. W. A. Sutherland, Path-components of function spaces, Quart. J. of Math. (Oxford) (2) 34 (1983), 223-233.

16. C. T. C. WALl, Finiteness conditions for CW-complexes, Ann. of Math. (2) 81 (1965), 56-69.

Department of Mathematics

The Edward Wright Building

Dunbar Street

ABERDEen AB9 2TY

SCOTLAND
New College

OXFORD 0X13BN

ENGLAND 